\title{
A telematics solution for the remote follow-up of malnourished children in underserved areas
}

\author{
Sury Bravo, Miguel A. Valero, José R. Blandino and Mario Ruiz
}

\begin{abstract}
The identification of malnourished children living under extreme poverty conditions in isolated areas is crucial to trigger urgent interventions like supplementary or therapeutic feeding. This work aims to strengthen the task of following-up malnourished maternal-child population in rural areas of developing countries like Nicaragua. The solution facilitates low-cost health nutritional remote monitoring to support rural communities at the point of care. Thus, the system allows medical staff to communicate with brigades, who transmit anthropometric measurements, such as weight and height of the children, from communities which are sited about $12 \mathrm{~km}$. far away. A hybrid WiMAX/WiFi architecture was deployed to provide affordable communications between the isolated communities and the health center. Furthermore, a free PBX software and an open information system, installed at the health center, support WiFi based mobile communications and information management to support the care needs of maternal-child population at risk.
\end{abstract}

\section{INTRODUCTION}

G LOBALLY more than one third of child deaths are caused by under nutrition; and the population living under poverty conditions in remote areas of developing countries are the first victims. Poverty conditions do impact on the expected child development. World Health Organization (WHO) states that over 200 million children under five years old fail to reach their full cognitive and social skills. Several efforts try to achieve this goal, e.g. to promote healthy children and families through appropriate facilities and effective combination of human resources and sustainable supporting technologies. Nevertheless, the identification of children who live under extreme poverty conditions and the deployment of an urgent intervention, such as supplementary or therapeutic feeding, is an open issue. Hence, a mHealth remote monitoring system has been implemented and deployed in Cusmapa, Nicaragua, to provide health personnel and health authorities with access to valuable information about growth and development of the children. Thus, the resulting system supports community information and affordable communication facilities whose aim is to monitor the status of disease and under nutrition of the maternal-child population at the point of need.

The validated mHealth remote monitoring system allows health volunteers in rural communities to acquire the weight and height of the children at the Point of Care. Information is gathered with low-cost mobile devices that submit data to a health information system using an ad hoc WiFi/WiMAX telecommunication network. The connectivity through WiFi and WiMax is a good alternative to GSM when there is not coverage or cost barriers arise. We propose this solution to overcome barriers such as lack of budget and mobile coverage so that no obstacles arise to collect the information. The information can be used not only to support local decisions about the nutrition and health of the unassisted children but also to reinforce strategic decisions that should be taken by higher health authorities. In this mode, the mHealth system described in this research paper contributes to support and strengthen medical and public health practice thus benefitting the most vulnerable population. This work also remarks the potential of the mHealth technologies validated at this research to provide information aimed to support decisions-making related with the delivering of timely health care for isolated populations.

The remainder of this paper is structured as follows: section two discusses the related works about the impact of mobile technologies in different health projects in developing countries. Section three details the health system profile of Nicaragua. Section four details the system architecture and section five covers the deployment and validation phases and finally we conclude this paper presenting discussion and future research.

\section{RELATED WORKS}

The increasing deployment of mobile communications was widely reported in 2007 by United Nations Foundation. It is especially focused on the potential of mobile health solutions to spread effective care services in low and middle income countries [1]. The expected benefits of mHealth, such as the promotion of quality of life related to health or timely monitoring of both diseases and therapies; rely on suitable deployments in tight cooperation with local communities [2]. According to the MDGs, any contribution of mHealth should take into account the situation of poverty and fragility of the underserved population; and then fostering a real empowerment at middle term. Lessons learned show that rural connectivity for mHealth monitoring 
services should not depend on population incomes. This promote the integration of free radio technologies, such as WiFi or open WiMax to reduce mobile communication costs if some initial funding is available.

Recent reports of mobile applications for the health Sector [1], [10] evidence the potential of mobile communications to radically improve healthcare services even in some of the most remote places and resource-poor population. In this sense, the available literature identifies a number of areas where mobile technologies potentially can make the difference in favor of maternal and child health [5].

A remarkable experience was carried out by Kalach et al. [6] who proposed using SMS technology to help community health workers to monitor antenatal care and track pregnant women by managing women at risk in the Musanze District of Rwanda. Ongoing evolution from SMS to IP based technologies in mobile and wireless communications may reduce data transmission delays and costs, fostering new initiatives for neonatal and perinatal care.

Other works, describe how mobile technologies improved mechanism for data collection and management. Kigali et al. [7] details an application of smart phone to improve antenatal care and expanded program on immunization services for the under-served population. The application has been integrated into the existing Healthcare Clinic Information System which is used by health centers across country. This is crucial to deploy solutions in developing countries because of it does not change the routine of healthcare workers in performing their duties. However the cost of the maintenance is a handicap as is difficult to get local level resources and in most cases resources has a poor and limited quality.

Several $\mathrm{mHealth}$ projects are playing an important role to support health needs in developing regions [8],[9],[11]. They contribute to the achievement of the health related MDGs by improving quality and timely delivery healthcare services and strengthening local capabilities.

\section{HEALTH SYSTEM PROFILE AND DEMOGRAPHIC CONTEXT OF NICARAGUA}

Human Development Index (HDI - 2011) of United Nations shows that Nicaragua is included in the medium human development group of countries. Nicaragua is ranked 129 with a HDI value of 0.589 . Nearly half $(42.4 \%)$ of the country's population is located in rural areas and suffers from both inadequate health care facilities and medical practitioners. The target areas of this research are the Chorotega region of San José de Cusmapa located at department of Madriz to 274 kilometers from the capital, Managua. The municipality encompasses 3 urban areas and 7 micro-regions that group 26 communities and 7072 inhabitants with $80 \%$ of rural population. Most of the population spends over 80 minutes to reach the health center by car or 6 hours by foot.

The coverage of mobile networks is under $30 \%$ and it is focused in the small village where a local health care center supports the area of $129.9 \mathrm{~km}^{2}$. San Jose de Cusmapa has a high rate of chronic severe malnutrition and stunting. The main causes of death among children under-5 year in poor and indigenous communities are malnutrition, diarrhea, respiratory diseases and meningitis [4].

The Ministry of Health of Nicaragua (MINSA) is the country's main supplier of health services via network of primary and secondary services. MINSA's first level of care consists of health care center usually located at municipality and the point of care (PoC) named rural health post located at rural communities. The PoC have lower response capacity than health center. Usually $\mathrm{PoC}$ is staffed by the volunteers of the community and depends on the health center for severe case referral, pharmaceutical deliveries, epidemiological management, and coordination of the health general activities. The information is manually updated and as a result of the widespread absence of communication systems it is based on paper reports. Therefore when health personnel need to collect health information, they must travel long journeys by foot, on horseback or by vehicles.

\section{SOLUTION FOR THE REMOTE FOLLOW-UP OF MALNOURISHED CHILDREN}

The solution provides to the community agents (brigades and midwives) at PoC El Carrizo with technological facilities to share information and support with the medical staff at San José de Cusmapa. It is important to point out that in PoCs the access to health care professionals is limited. El Carrizo PoC hardly has medical supplies and covers almost two thousand people including the seven surrounding communities (Las Jaguas, Jocomico, El Gavilán, El Ángel III, El Naranjo, El Jobo, Aguas Calientes and la Jabonera).

The complexity of this scenario requests both a voice communication solution between the rural health post and the health center but also a low cost solution to acquire the weight and height of the children. This integrated health monitoring system should be able to provide authorized staff with updated reports about antenatal monitoring tasks, health and nutritional status of the communities, malnutrition, immunization control and regular communications. The importance of data updated by health care staff is crucial to timely detect the source of problem to implement measures addressed to alleviate and improve health status population permanently.

\section{A. System Architecture}

As Figure 1 shows, to deploy a mobile devices based architecture a combination of data communication links was designed. Brigades hold a medium price mobile device that interacts through a Bluetooth connection with a low cost Nintendo Wii Balance board and WiiMote to acquire weight and height of the children. Once children data is captured, the mobile connects via WiFi and WiMax with the local Health Center to store the data in the Health Information Station or to communicate with the physician.

The WiMax base station installed in the health center of San José de Cusmapa is a Breeze Access VL unit. The transmitter was connected to a $120^{\circ}$ sectorial antenna for 5.8 
$\mathrm{GHz}(15 \mathrm{dBI})$ that covers an area of about 100 square kilometers where communities are placed. The installation was placed on a tower at $15 \mathrm{~m}$. height thus overpassing surrounding trees and point to El Carrizo primary school sited $7.4 \mathrm{~km}$. in Line of Sight. The subscriber unit at the rural health post is a Breeze Access VL SU-ODU which also provides an IEEE $802.11 \mathrm{~b} / \mathrm{g}$ link $30 \mathrm{~m}$. around. Thus, the brigades or teachers at the local school can use a mobile phone supporting VoIP and SIP protocol (Trials were done with Nokia E51, Nokia E65) to contact with medical staff who uses another mobile phone connected by WiFi to the Alvarion $\mathrm{Wi}^{2}$ Extender installed in San José de Cusmapa. We also developed a health geographical information system implemented with gvSIG 1.9, an open tool that was fed with GPS data acquired at the communities, which permitted to provide a data base with four information layers: fertile women, children, people with disabilities and diseases.

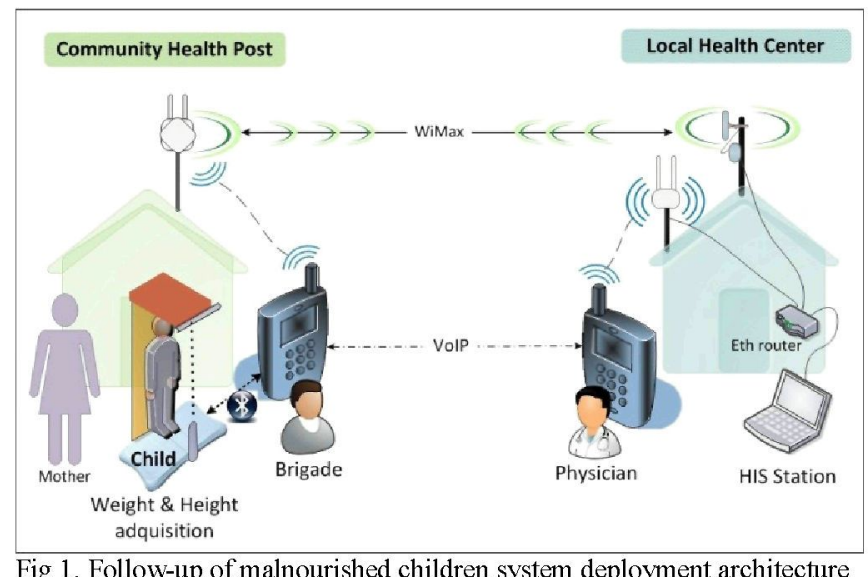

The Bluetooth 2.1 interaction between the Nintendo Wii Balance board, the WiiMote and the mobile device requested the implementation of a measuring interface using Wiiuse, a free library written in C. Next, a Java wrapper called Wiiusej made possible the interface with Android NDK by using JNI to interact between Java and $C$ libraries. Thus, the measuring system can be easily controlled with an Android 2.1 low cost mobile phone such as the Huawei u8110 tested in the trials. The accuracy of the weighting system is about 10 grams thanks to the calculation of weight as the medium value of four sensors. The measurement of the height of the children is performed by calculating the distance between a WiiMote placed on the ground and a sensors bar placed over the traditional measuring tool (horizontal and vertical view angle and distance between two infrared led).

\section{VALIDATION SCENARIOS AND RESULTS}

The use case has been validated along three incremental stages. Firstly, the community geographical information system was integrated in July, 2009 followed by training sessions with the local authorities. After one year of deployment, full data from four pilot communities was gathered: Angel 3 (321 inhabitants and 58 houses), Apante (398 inhabitants and 63 houses), Jaguas (255 inhabitants and
42 houses) and Roble (321 inhabitants and 53 houses). The usability of the system allowed acquiring the data of a total of 1295 inhabitants distributed in 221 households place on the four priority communities addressed by the health care center. The local criteria for the research was to select two distant communities which are placed about $12 \mathrm{~km}$. from Cusmapa and two closer ones which are placed about $5 \mathrm{~km}$. away but with mountain access.

A second validation phase of 12 months was started once the full communication system was installed in August 2010. After that, operational results showed the reliability of WiMAX and WiFi links as well as current short periods of power outages may not succeed. Although the health care center has local energy, an Uninterruptible Power Supply was placed to prevent from periodic supply failures. Power supply at El Carrizo health post and school is provided through solar cells. Two physicians, six nurses and three community actors reported very good quality in the VoIP radio links as tested in field trial conditions.

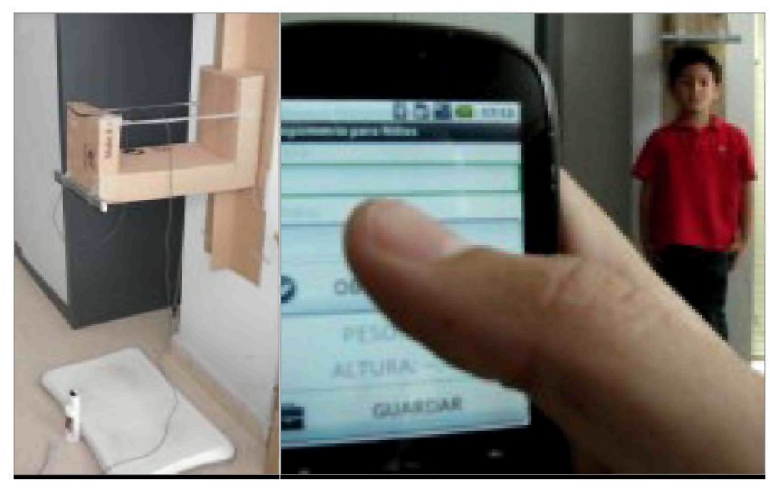

Fig. 2. Verification of weight and height acquisition system

Finally, the weight and height acquisition system has been also tested by paying attention to the reliability of the Bluetooth connection, the usability of the user interface, the quality of the measurements and the acceptance from eight people without previous knowledge of Android systems.

The most difficult was found when Bluetooth connection between de devices is interrupted to save battery. This problem led to provide users with some connection instructions through the mobile user interface as well as to introduce a software session manager to avoid device interruptions between measurement changes. Figure 2, shows the verification tests. The left part of the figure is the original prototype where the Wii Balance Board is placed on the ground for weight acquisition and the sensors bars is adapted to take height. The test about acquisition the weight and height was carried out with real users provided information about their anthropometric measurements. Eight children of the El Naranjo community have taken part in this stage such as shows Table 1. This table shows a comparison between the measurements made by Medical Brigade with the measurements obtained by the monitoring system. Some differences between the manual and automatic process appear as shown by Figure 3. First, the accuracy of the weighting system has been decreased because of Wii 
balance board needs a level surface; and this requirement was difficult to provide during the test carried out in the $\mathrm{PoC}$ in the El Naranjo community.

TABLE 1

MEAStrREMENTS OF WEIGHT COMPARISON

\begin{tabular}{|c|c|c|c|c|}
\hline \multirow{2}{*}{ Gender } & \multirow{2}{*}{$\begin{array}{c}\text { Age } \\
\text { months/years }\end{array}$} & \multicolumn{2}{|c|}{$\begin{array}{l}\text { Medical Brigade } \\
\text { Measurements }\end{array}$} & \multirow{2}{*}{$\begin{array}{c}\text { System Monitoring } \\
\text { Measurements } \\
\text { UPM Weight } \\
(\mathrm{kg})\end{array}$} \\
\hline & & $\begin{array}{c}\text { MBM } \\
\text { Weight }(\mathrm{kg})\end{array}$ & $\begin{array}{c}\text { MBM } \\
\text { Height }(\mathrm{cm})\end{array}$ & \\
\hline $\mathrm{M}$ & $17 \mathrm{~m}$ & 8,1 & 75,2 & 0 \\
\hline F & $17 \mathrm{~m}$ & 7,5 & 73,7 & 0 \\
\hline $\mathrm{F}$ & $6 y$ & 17 & 100 & 16,5 \\
\hline $\mathrm{F}$ & $5 y$ & 13,7 & 90,7 & 13,2 \\
\hline $\mathrm{F}$ & $6 y$ & 16,5 & 104,8 & 15,7 \\
\hline M & $5 y$ & 20,9 & 113 & 20 \\
\hline M & $5 y$ & 18,4 & 101 & 17,5 \\
\hline M & $5 y$ & 14,2 & 95 & 14 \\
\hline
\end{tabular}

Additionally this requirement obstructed the measurement of the height, because of the field of view of the camera is fairly restricted and hence it must be pretty much directly in front of the sensor bar for this to work. The PoC is a rural house under poor conditions, used by health staff to provide health services to isolated population. Second, in the column weight of the automatic system the data that appears with zero corresponds those children under two years which was difficult to maintain unmoving on the Wii balance board during a short time.

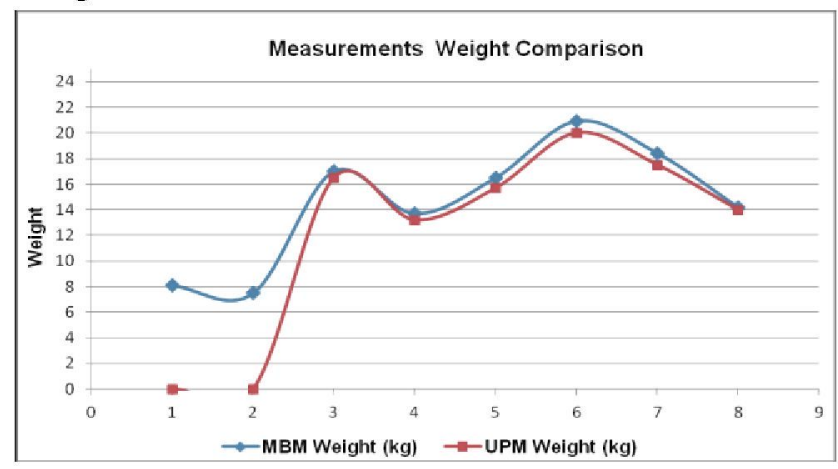

Fig. 3. Measurement comparison of automatic and manual weight capture

Moreover, the solution was presented at the health center and the nurses think that could be a useful tool in order to significant reduction in data transmission delay compared to current paper-based system and even increase data quality reported by the surveys or nutritional controls.

\section{CONCLUSION AND FUTURE RESEARCH}

The deployment experience acquired along the last two years of the system validation leads to conclude that this mHealth remote monitoring system is accepted by health care staff as it takes into account their practical care needs and organizational procedures. Although the medical doctors agreed a weekly routine with to monitor the health state of the communities, higher use is expected at emergency situations. The main reason for this is the work overload at San Jose de Cusmapa health center that makes prevention activities to be held in background. The weight and height acquisition system is positively evaluated by the nurses in order to save time when displacing to local communities to get children data. The head of the health care center expects to enhance information management to perform acuter epidemiologic studies in the area.

Future research is planned to extend the WiMax/WiFi subscriber units to El Roble and El Apante communities which also belong to Cusmapa area. Emerging Android compatible low cost mobile devices, made by Samsung, HTC and Motorola, are been tested for battery capacity reasons. Although solar power is used to charge the devices, its self-autonomy is critical for higher success with the measuring system. In addition, a new web interface is being validated at the university lab to communicate San José de Cusmapa health information system with the head of area sited in Somoto, which is in charge of the department epidemiological studies.

\section{ACKNOWLEDGMENT}

We would like to thank the health care staff at San Jose de Cusmapa center and Vodafone (José Manuel Azorín) for their professional support to this project. This research was partially funded by the Programme "Comunidades Rurales del Milenio-UPM". Thanks to Eng. David Estivariz, who implemented the weighting system. Authors thank the anonymous reviewers for their valuable comments.

\section{REFERENCES}

[1] UN Foundation, Vodafone Foundation Partnership, "mHealth for development: The opportunity of mobile technology for healthcare in the developing world," Tech. Rep. Final Report, 2009.

[2] World Health Organization, "Country Cooperation Strategy at a Glance (Final Report)", 2006. Available: http://www.paho.org/english/d/csu/BriefNICEng.pdf

[3] World Health Organization, "Early child development: A powerful equalizer," Tech. Rep. Final Report, 2007.

[4] Pan American Health Organization. "Health systems profile in Nicaragua: Monitoring and analyzing health systems Change/Reform", Washington D.C., 2009.

[5] C. Zhenwei, M. Yamamichi, V. Hausman and D. Altman, "Mobile applications for the health sector," World Bank, Dec. 2011. 2011.

[6] J. Kalach, "Saving mothers and newborn lives in the community using rapidSMS technology-experience from Musanze District of Rwanda," presented at the International Conference on Community Health, Kigali, Rwanda.

[7] J. Kaewkungwal, et al. (2010, November), "Application of smart phone in "Better Border Healthcare Program": A module for mother and child care,". BMC Medical Informatics and Decision Making [open access]. Volume 10(69), 1-12. Available: http://www.biomedcentral.com/content/pdf/1472-6947-10-69.pdf

[8] A. Fleishman, et al. (2010, spring), "Validation process for a social entrepreneurial telemedicine venture in east Africa,". International Journal for Service Learning in Engineering: Humanitarian Engineering and Social Entrepreneurship [open access]. Volume 5(1), 1-24. Available:

http://ibrary.queensu.ca/ojs/index.php/ijsle/article/view/2344/2469

[9] T. Tamrat and S. Kachnowski, "Special Delivery: An Analysis of mHealth in Maternal and Newborn Health Programs and their Outcomes around the World," Maternal Child J., vol. 16, pp. 1-10, July 2011.

[10] Novartis Foundation for Sustainable Development, "E-health for development (Technical Report)," Annual report, 2011.

[11] M. Bogan, et al., "Improving standards of care with mobile applications in Tanzania (Online)," presented at the W3C Workshop on the Role of Mobile Technologies in Fostering Social and Economic Development in Africa, Maputo, Mozambique, April 1, 2009. 